\title{
Performance gain bounds of coherently combining multiple radars in a target-based calibration manner
}

\author{
LIU Xinghua*, XU Zhenhai, and XIAO Shunping \\ State Key Laboratory of Complex Electromagnetic Environment Effects on Electronics and Information System, \\ National University of Defense Technology, Changsha 410073, China
}

\begin{abstract}
To achieve a high signal-to-noise ratio (SNR) while maintaining moderate radar antenna, a target-based calibration manner is available to coherently combine multiple radars. The key to this calibration manner is to estimate coherence parameters (CPs), i.e., time and phase calibration values in transmission and reception estimation, by separating the target returns into monostatic and bistatic echoes. However, CPs estimations exist uncertainties, which will affect the performance gain after multiradar coherent combination. The principle of coherently combining multiple radars is elaborated and the signal probability model for CPs estimation is established. On this basis, CPs Cramer-Rao bound (CRB) is derived in the closed-form, according to which the non-tight and tight upper bounds for multiple radars coherent combination performance gain are derived in the closed-form and via Monte Carlo (MC) simulations, respectively. Simulations validate the correctness of the derived CRB and gain bounds.
\end{abstract}

Keywords: coherently combining multiple radars, Cramer-Rao bound (CRB), coherence gain.

DOI: $10.21629 /$ JSEE.2019.02.07

\section{Introduction}

A single radar has a theoretical maximum signal-to-noise ratio (SNR) for a given size target at a given range, which directly affects the ability of the radar to detect, track, and identify the target [1]. Since the SNR of a radar is proportional to the square of the antenna aperture, the traditional quest for enhancing the SNR is to develop the large aperture radar, e.g., the ground-based radar prototype (GBRP) and the sea-based X-band radar (SBX). However, developing a large aperture radar system is costly and not easily transportable, which seriously restricts the survivability and flexibility of this type of the radar. Alternatively, by jointly adjusting the transmitting/receiving time and phase of several co-located small radars, it is possible to combine

\footnotetext{
Manuscript received November 07, 2017.

*Corresponding author.

This work was supported by the National Natural Science Foundation of China (61471372).
}

them into a coherently functioning system for obtaining significantly the enhanced SNR [1-4]. By doing this, the combined radar system will be benefitial to the transportability and economic advantage, while maintains the comparable echo SNR to the large aperture radar. When $N$ identical radars are coherently combined, an $N^{3}$ SNR gain over a single radar can be acquired [5].

Actually, due to the distributed arrangement of radars, it cannot coherently combine multiple radars directly. The necessary time and phase adjustments in transmission and reception should be carried out to calibrate the decoherence induced by the disparate propagation path and the synchronization error. If the relative separations among radars are accurately known, according to which time and phase calibrations can be calculated and then compensated. The corresponding radar technique is well-known under the name phased-array radar [6]. However, measuring the relative positions is difficult and subjects to the increasing errors as the separation of radar antennas increases, if radars are not relatively fixed [1].

For this reason, an approach for coherently combining multiple radars in a target-based calibration manner was proposed in [2,3]. This approach relies on estimating coherence parameters (CPs), i.e., time and phase calibration values in transmission and reception, by transmitting orthogonal waveforms and separating the target returns into monostatic and bistatic echoes. In this way, the relative separations among radars are not critical anymore. Inspired by this approach, relevant works are springing up. In [7], a clean signal reconstruction approach was proposed to solve the bias in this CPs estimation due to transmitting non-ideally orthogonally waveforms. A generalize dualradar coherently combining paradigm was proposed in [8], which clearly explained how to coherently combine dualradar based on a moving target. In [9-11], a dual-radar coherently combining system based on step frequency signal was proposed to enhance the system robustness to time synchronization error. Several dual-radar coherently com- 
bining experiments can be found in [12-14].

Throughout the existing research, most of them focus on how to coherently combine multiple radars. One important problem is neglected. Although the target-based manner does not require precise relative separations among radars, it has to estimate CPs. Due to the existence of the measurement noise, the estimated CPs are uncertain. To adjust the time and phase in transmission and reception based on these uncertain CPs, the anticipate performance gain cannot be obtained. Therefore, it is necessary to analyze the performance gain bound under the given noise condition, including the factors affecting the performance gain bound. All these above motivate the investigation of this paper. Similar works could be found in $[15,16]$, but their efforts focused on the architecture which radar transmitters and receivers were separated, whose conclusions were less instructive for multiple radars coherent combination.

The major contributions of this paper are outlined as follows:

(i) Based on the principle of multi-radar coherent combination in the target-based calibration manner, the signal probability model for CPs is established. On this basis, CPs Cramer-Rao bounds (CRBs) are derived in the closedform.

(ii) The non-tight and tight upper performance gain bounds for multiple radars coherent combination are derived in the closed-form and via Monte Carlo (MC) simulations, respectively.

The rest of this paper is organized as follows. Section 2 introduces the principle of coherently combining multiple radars. In Section 3, the signal probability model for the CPs estimation is established. Section 4 derives the CRB of CPs. The performance gain bounds are analyzed in Section 5. Conclusions are drawn in Section 6.

\section{Principle of coherently combining multiple radars}

For distributed deployment radars, due to disparate propagation paths from radars to target, synchronization errors, and the target scattering characteristics difference, the signal decoherence phenomena occur in both transmission and reception. Namely, radars transmitted signals are mutually incoherent at the target (decoherence-on-transmit), and so do echoes received by each radar (decoherence-onreceive). Apparently, the anticipated coherent gain cannot be obtained without reasonable calibration. If radars are co-located $[17,18]$, each radar will exhibit the same perspective and scattering characteristics for a given target. Then, the signal decoherence only comes from disparate propagation paths and synchronization errors, which can be represented by time misalignment and phase offset in the signal.
Therefore, to coherently combine multiple radars, i.e, achieve both coherent-on-transmit and -receive, time and phase adjustments should be carried out to calibrate the signal decoherence. Put it more concretely, if the signal transmitted by radar 1 (reference radar) arrives at the target after the signal transmitted by radar 2, proper time and phase adjustments for radar 2 are needed to slow down and retard its transmitting time and phase appropriately. Such calibration processes can be formulated as follows [7,8].

Let $s\left(t-\kappa_{k}\right) \mathrm{e}^{j 2 \pi f_{c}\left(t-\kappa_{k}\right)+j \varphi_{k}}(k=1, \ldots, N)$ denote the transmitted signal of radar $k, s(t)$ is the common baseband signal (identical waveform), $\kappa_{k}$ is the time synchronization error of radar $k$ compared to the reference clock, and $\varphi_{k}$ is the initial phase of radar $k$. Then, these transmitted signals propagated to the target can be expressed as

$$
s\left(t-\kappa_{k}-\tau_{k}\right) \mathrm{e}^{j 2 \pi f_{c}\left(t-\kappa_{k}-\tau_{k}\right)+j \varphi_{k}}, \quad k=1, \ldots, N
$$

where $f_{c}$ is the carrier frequency, $\tau_{k}=R_{k} / c$ denotes the propagation time from radar $k$ to the target, $R_{k}$ is the propagation range related to $\tau_{k}, c$ is the speed of light.

Apparently, from (1), radars transmitted signals are mutually incoherent at the target, manifested as misaligned time and phase offsets. To multiply the radio frequency (RF) electric field impinging upon the target, i.e., achieve coherent-on-transmit and obtain the coherence gain, fine transmitting time and phase adjustment are necessary. Without loss of generality, assume radar 1 is the reference radar, it is essential to adjust the transmitting time and phase of other radars. The adjusted signals can be expressed as $s\left(t-\kappa_{k}+\Delta \tau_{k}^{t}\right) \mathrm{e}^{j 2 \pi f_{c}\left(t-\kappa_{k}\right)+j \varphi_{k}} \mathrm{e}^{-j \Delta \phi_{k}^{t}}$, where

$$
\begin{gathered}
\Delta \tau_{k}^{t}=\left(\tau_{k}-\tau_{1}\right)+\left(\kappa_{k}-\kappa_{1}\right) \\
\Delta \phi_{k}^{t}=-2 \pi f_{c}\left[\left(\tau_{k}-\tau_{1}\right)+\left(\kappa_{k}-\kappa_{1}\right)\right]+\left(\varphi_{k}-\varphi_{1}\right)
\end{gathered}
$$

are the transmit time and phase calibration values (transmit CPs) of radar $k$. The pictorial illustration of transmit decoherence and correction is shown in Fig. 1.

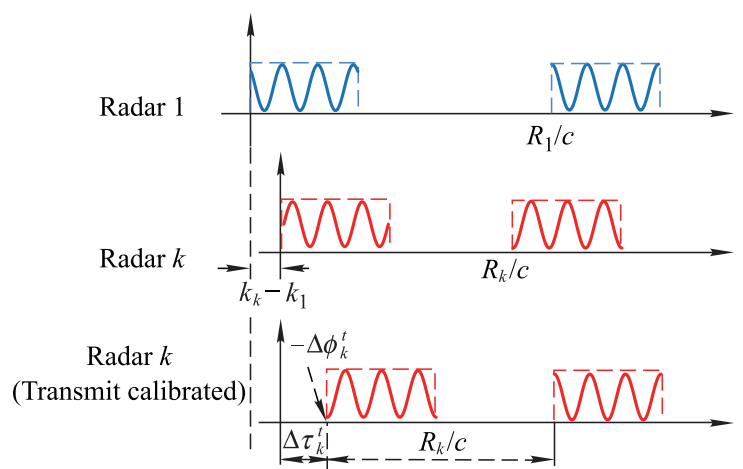

Fig. 1 Transmit decoherence and correction

Let $u(t) \mathrm{e}^{j 2 \pi f_{c} t}$ denote the reflected echo at the target, and $u(t)$ is the complex envelope. Then the echo received 
by radar $l$ after down-conversation is (assuming each radar can realize the coherent reception)

$$
u\left(t-\tau_{l}+\kappa_{l}\right) \mathrm{e}^{-j 2 \pi f_{c} \tau_{l}+j 2 \pi f_{c} \kappa_{l}-j \varphi_{l}}, \quad l=1, \ldots, N .
$$

Similarly, to achieve the coherent-on-receive, appropriate time and phase shifts are also necessary for the echoes received by the radars except radar 1 . The adjusted echo is $u\left(t-\tau_{l}+\kappa_{l}+\Delta \tau_{l}^{r}\right) \mathrm{e}^{-j 2 \pi f_{c} \tau_{l}+j 2 \pi f_{c} \kappa_{l}-j \varphi_{l}} \mathrm{e}^{-j \Delta \phi_{l}^{r}}$, where

$$
\begin{gathered}
\Delta \tau_{l}^{r}=\left(\tau_{l}-\tau_{1}\right)-\left(\kappa_{l}-\kappa_{1}\right) \\
\Delta \phi_{l}^{r}=-2 \pi f_{c}\left[\left(\tau_{l}-\tau_{1}\right)-\left(\kappa_{l}-\kappa_{1}\right)\right]-\left(\varphi_{l}-\varphi_{1}\right)
\end{gathered}
$$

denote the receive time and phase calibration values (receive CPs) of radar $l$. The superscript $t$ is the abbreviation for "transmit", while the superscript $r$ is the abbreviation for "receive". The pictorial illustration of receive decoherence and correction is shown in Fig. 2.

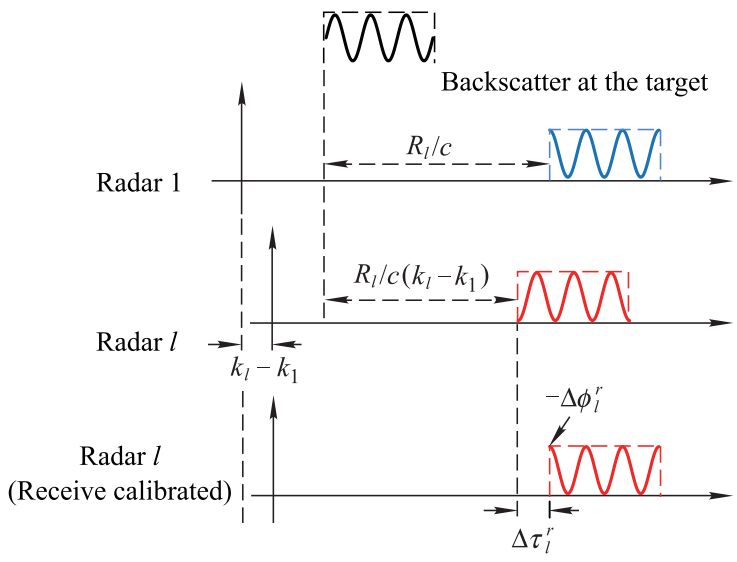

Fig. 2 Receive decoherence and correction

\section{Signal probability model for CPs estimation}

From the analyses above, the essence of multiple radar coherent combining is to adjust the transmitting and receiving time and phase based on CPs. According to the definition of CPs in (2) and (4), CPs are the relative time/phase relationship between signals. Particularly, $\Delta \tau_{1}^{t}=\Delta \tau_{1}^{r}=$ $\Delta \phi_{1}^{t}=\Delta \phi_{1}^{r}=0$, and the remaining unknown CPs are $\left\{\Delta \tau_{k}^{t}, \Delta \tau_{l}^{r}, \Delta \phi_{k}^{t}, \Delta \phi_{l}^{r}\right\}(k, l=2, \ldots, N)$. For simplicity, $\left\{\Delta \tau_{k}^{t}, \Delta \tau_{l}^{r}\right\}$ and $\left\{\Delta \phi_{k}^{t}, \Delta \phi_{l}^{r}\right\}$ are collectively called the time and phase CPs, respectively.

Unlike the phased array radar, CPs between subarrays (radars) are usually known as a priori. The target-based calibration manner provides another approach to coherently combine distributed radars, i.e., separate target returns to estimate CPs and then calibrate the decoherence via the CPs estimation. Let $y_{l k}(t)(k, l=1, \ldots, N)$ denote the separated echo corresponding to the propagation path from radar $k$ to radar $l$. Then, $\left\{y_{l k}(t), k=l\right\}$ is the monostatic echo, and $\left\{y_{l k}(t), k \neq l\right\}$ is the bistatic one (as shown in Fig. 3). By analyzing the mutual time and phase relationship of echo pairs properly selected from the separated echoes, CPs can be estimated.

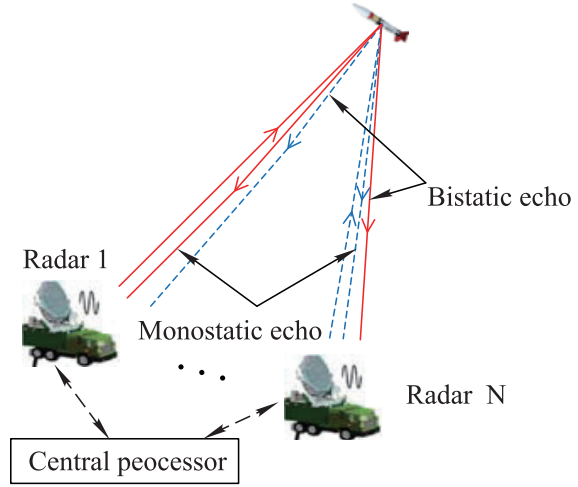

Fig. 3 Separated echoes when multiple radars transmit orthogonal waveforms

For example, if the echo pair $\left\{y_{l 1}(t), y_{l k}(t)\right\}$ is selected, their receive paths are identical, while transmit paths are not. Then, the time and phase differences between this echo pair are the transmit CPs of radar $k$. Similarly, other CPs can be estimated. That is, when multiple radars transmit orthogonal waveforms, the complete information of CPs is contained in target returns.

Although the above estimation strategy can obtain CPs observations, these observations are inaccurate due to the influence of the measurement noise. The uncertainty of CPs estimates will definitely affect the performance of the multi-radar coherent combination. Therefore, it is necessary to evaluate the estimation performance under noisy conditions.

A common tool for evaluating the estimation performance under the noise is the CRB, which can be derived under the reasonable probability model assumption [19]. Assume there is a point target in the space, for this given CPs estimation problem, the baseband probability model for target return received at radar $l$ is

$$
r_{l}(t)=\sum_{k=1}^{N} \xi^{\prime} s_{k}\left(t-\tau_{l k}\right) \mathrm{e}^{-j 2 \pi f_{c} \tau_{l k}+j \varphi_{k}-j \varphi_{l}}+w_{l}(t)
$$

where $\xi_{l k}^{\prime}$ denotes the scattering coefficient of the $k l$ th propagation (if distributed radars are co-located, $\xi_{l k}^{\prime} \approx \xi^{\prime}$ ); $\left\{s_{k}(t), k=1, \ldots, N\right\}$ is the orthogonal waveform set, where $\int_{T}\left|s_{k}(t)\right|^{2} \mathrm{~d} t=1$, and $T$ is the common duration of all transmitted waveforms; $\tau_{l k}=\tau_{k}+\tau_{l}+\kappa_{k}-\kappa_{l}$ denotes the propagation time from radar $k$, to target and to radar $l$; $w_{l}(t)$ is the additive noise introduced during the radar $l$ reception, which is assumed to be a zero-mean, independent and identically distributed (i.i.d), complex white Gaussian 
random process with autocorrelation function $N_{0} \delta(\tau), N_{0}$ is the power spectral density (PSD).

For the established probability model in (5), all of the radars have the same transmission power and noise assumption. That is, the SNR of all radars are identical. Therefore, in the following, we use a uniform SNR instead of distinguishing the SNRs of different radars.

Let $\xi=\xi^{\prime} \mathrm{e}^{-j 2 \pi f_{c} \tau_{11}}$, (5) can be further written as

$$
r_{l}(t)=\xi \sum_{k=1}^{N} s_{k}\left(t-\tau_{l k}\right) \mathrm{e}^{j \Delta \phi_{k}^{t}+j \Delta \phi_{l}^{r}}+w_{l}(t) .
$$

In our paper, $\xi$ is treated as an unknown complex amplitude term containing the target impact and additional phase offset. In the following, for convenience, we denote $\xi=\xi^{R}+j \xi^{I}$. Furthermore, combining (2), (4) and the definition of $\tau_{l k}$, one obtains

$$
\begin{aligned}
& \Delta \widehat{\tau}_{k}^{t}=\frac{1}{N} \sum_{l=1}^{N}\left(\tau_{l k}-\tau_{l 1}\right), \quad k=2, \ldots, N, \\
& \Delta \widehat{\tau}_{l}^{r}=\frac{1}{N} \sum_{k=1}^{N}\left(\tau_{l k}-\tau_{1 k}\right), \quad l=2, \ldots, N .
\end{aligned}
$$

\section{CPs CRB}

Under suitable regularity conditions, CRB provides a lower bound for the variance of any unbiased estimate. Given an unknown vector $\boldsymbol{\vartheta}$, constituted of elements $\vartheta_{i}$, the bound can be formulated as [19, 20]

$$
\operatorname{var}\left(\widehat{\vartheta}_{i}\right) \geqslant\left[\boldsymbol{J}^{-1}(\boldsymbol{\vartheta})\right]_{i i}
$$

where $\widehat{\boldsymbol{\vartheta}}$ is the unbiased estimate of $\boldsymbol{\vartheta} ;\left[\boldsymbol{J}^{-1}(\boldsymbol{\vartheta})\right]_{i i}$ is the $i$ th element in $\boldsymbol{J}^{-1}(\boldsymbol{\vartheta}) . \boldsymbol{J}(\boldsymbol{\vartheta})$ is the fisher information matrix (FIM), which is given by

$$
\boldsymbol{J}(\boldsymbol{\vartheta})=-E_{\boldsymbol{r} \mid \boldsymbol{\vartheta}}\left\{\nabla_{\boldsymbol{\vartheta}}\left[\nabla_{\boldsymbol{\vartheta}} \ln p(\boldsymbol{r} \mid \boldsymbol{\vartheta})\right]^{\mathrm{T}}\right\}
$$

where $p(\boldsymbol{r} \mid \boldsymbol{\vartheta})$ is the joint probability function (PDF) of $\boldsymbol{r}$ conditioned on $\vartheta, E_{\boldsymbol{r} \mid \boldsymbol{\vartheta}}\{\cdot\}$ denotes the expectation with respect to $p(\boldsymbol{r} \mid \boldsymbol{\vartheta})$. The FIM can also be defined as the complex FIM, which can simplify the computational complexity. Details are shown in [21].

In our case, CPs in conjunction with the complex amplitude term $\xi$ are the unknown parameters to be estimated, which can be integrated into the following vector:

$$
\boldsymbol{\vartheta}=\left[\left(\Delta \boldsymbol{\tau}^{t}\right)^{\mathrm{T}},\left(\Delta \boldsymbol{\tau}^{\boldsymbol{r}}\right)^{\mathrm{T}},\left(\Delta \boldsymbol{\phi}^{t}\right)^{\mathrm{T}},\left(\Delta \boldsymbol{\phi}^{\boldsymbol{r}}\right)^{\mathrm{T}}, \xi^{R}, \xi^{I}\right]^{\mathrm{T}}
$$

where $\boldsymbol{\vartheta} \in \mathbf{R}^{(4 N-2) \times 1}$ and

$$
\begin{gathered}
\Delta \boldsymbol{\tau}^{t}=\left[\Delta \tau_{2}^{t}, \ldots, \Delta \tau_{N}^{t}\right]^{\mathrm{T}} ; \Delta \boldsymbol{\tau}^{\boldsymbol{r}}=\left[\Delta \tau_{2}^{r}, \ldots, \Delta \tau_{N}^{r}\right]^{\mathrm{T}} \\
\Delta \boldsymbol{\phi}^{t}=\left[\Delta \phi_{2}^{t}, \ldots, \Delta \phi_{N}^{t}\right]^{\mathrm{T}} ; \Delta \boldsymbol{\phi}^{\boldsymbol{r}}=\left[\Delta \phi_{2}^{r}, \ldots, \Delta \phi_{N}^{r}\right]^{\mathrm{T}} .
\end{gathered}
$$

According to (7) and (8), $\tau_{l k}$ is a function of the unknown time CPs, therefore the log-joint PDF of the observations conditional on $\boldsymbol{\vartheta}$ can be obtained from the Karhunen-Loéve expansion of $r_{l}(t)$ [22-24]

$$
\begin{gathered}
\ln p(\boldsymbol{r} ; \boldsymbol{\vartheta}) \propto \\
-\frac{1}{N_{0}} \sum_{l=1}^{N} \int_{T}\left|r_{l}(t)-\xi \sum_{k=1}^{N} s_{k}\left(t-\tau_{l k}\right) \mathrm{e}^{j \Delta \phi_{k}^{t}+j \Delta \phi_{l}^{r}}\right|^{2} \mathrm{~d} t
\end{gathered}
$$

where $\boldsymbol{r}=\left[r_{1}(t), \ldots, r_{N}(t)\right]^{\mathrm{T}}$ is the observations of multiple radars.

Since $p(\boldsymbol{r} \mid \boldsymbol{\vartheta})$ is a function of $\tau_{l k}$, by the vector parameter CRB transformations conclusion, the CRB inequality can be expressed in an alternative form [25]

$$
\operatorname{var}\left(\widehat{\vartheta}_{i}\right) \geqslant\left[\boldsymbol{P} \boldsymbol{J}^{-1}(\boldsymbol{\psi}) \boldsymbol{P}^{\mathrm{T}}\right]_{i i} .
$$

Or $\boldsymbol{J}^{-1}(\boldsymbol{\vartheta})=\boldsymbol{P} \boldsymbol{J}^{-1}(\boldsymbol{\psi}) \boldsymbol{P}^{\mathrm{T}}$, where

$$
\boldsymbol{\psi}=\left[\boldsymbol{\tau}^{\mathrm{T}},\left(\Delta \boldsymbol{\phi}^{t}\right)^{\mathrm{T}},\left(\Delta \boldsymbol{\phi}^{\boldsymbol{r}}\right)^{\mathrm{T}}, \xi^{R}, \xi^{I}\right]^{\mathrm{T}} .
$$

$\boldsymbol{\psi} \in \mathbf{R}^{\left(N^{2}+2 N\right) \times 1}, \boldsymbol{\tau}=\left[\tau_{11}, \tau_{12}, \ldots, \tau_{N N}\right]^{\mathrm{T}}, \boldsymbol{J}(\boldsymbol{\psi})$ is the FIM with respect to $\psi$, and $\boldsymbol{P}$ is the Jacobian matrix

$$
[\boldsymbol{P}]_{i, j}=\partial \boldsymbol{\vartheta}_{i} / \partial \psi_{j}
$$

From (7), (8) and (16), $\boldsymbol{P} \in \mathbf{R}^{(4 N-2) \times\left(N^{2}+2 N\right)}$ can be calculated as

$$
\boldsymbol{P}=\left[\begin{array}{cc}
\boldsymbol{H}_{2(N-1) \times N^{2}} & \boldsymbol{O}^{\mathrm{T}} \\
\boldsymbol{O}_{2 N \times N^{2}} & \boldsymbol{I}_{2 N \times 2 N}
\end{array}\right]
$$

where

$$
\begin{aligned}
& \boldsymbol{H}=\left[\begin{array}{l}
\boldsymbol{H}_{1} \\
\boldsymbol{H}_{2}
\end{array}\right]=\left[\begin{array}{llll}
\boldsymbol{H}_{11} & \boldsymbol{H}_{12} & \cdots & \boldsymbol{H}_{1 N} \\
\boldsymbol{H}_{21} & \boldsymbol{H}_{22} & \cdots & \boldsymbol{H}_{2 N}
\end{array}\right] \\
& \boldsymbol{H}_{1 n}=\frac{1}{N}\left[-\mathbf{1}_{(N-1) \times 1} \quad \boldsymbol{I}_{N-1}\right], \quad n=1, \ldots, N \\
& \boldsymbol{H}_{21}=-\frac{1}{N} \boldsymbol{I}_{(N-1) \times N} ; \boldsymbol{H}_{2 n}=\frac{1}{N}\left[\begin{array}{c}
\boldsymbol{o}_{(n-2) \times N} \\
\boldsymbol{1}_{1 \times N} \\
\boldsymbol{0}_{(N-n) \times N}
\end{array}\right], \\
& n=2, \ldots, N \text {. }
\end{aligned}
$$

$\boldsymbol{O}$ is the all-zero matrix, $\boldsymbol{1}$ is the all-one matrix, and $\boldsymbol{I}$ is the identity matrix.

Assume that transmitted waveforms are ideally orthogonal, namely $\int_{T} s_{k}\left(t-\tau_{l k}\right) s_{k^{\prime}}^{*}\left(t-\tau_{l^{\prime} k^{\prime}}\right) \mathrm{d} t=0$ holds for $l \neq l^{\prime}$ or $k \neq k^{\prime}$. Using (10) with $\boldsymbol{\psi}$ replacing $\vartheta$, it is not difficult to calculate each element in $\boldsymbol{J}(\boldsymbol{\psi})$. The detailed expression for each element in the $\operatorname{FIM} \boldsymbol{J}(\boldsymbol{\psi})$ is shown as follows:

$$
-E_{\boldsymbol{r} \mid \boldsymbol{\psi}}\left\{\frac{\partial^{2}}{\partial \tau_{l k} \partial \tau_{l^{\prime} k^{\prime}}} \ln p(\boldsymbol{r} \mid \boldsymbol{\psi})\right\}=
$$




$$
\begin{aligned}
& \left\{\begin{array}{l}
0, \text { others } \\
\frac{8 \pi|\xi|^{2}}{N_{0}} \beta_{k}^{2}, \quad l=l^{\prime}, k=k^{\prime}
\end{array}\right. \\
& -E_{\boldsymbol{r} \mid \psi}\left\{\frac{\partial^{2}}{\partial \tau_{l k} \partial \Delta \phi_{k^{\prime}}^{t}} \ln p(\boldsymbol{r} \mid \psi)\right\}=0 \\
& -E_{\boldsymbol{r} \mid \boldsymbol{\psi}}\left\{\frac{\partial^{2}}{\partial \tau_{l k} \partial \Delta \phi_{k^{\prime}}^{r}} \ln p(\boldsymbol{r} \mid \psi)\right\}=0 \\
& -E_{\boldsymbol{r} \mid \boldsymbol{\psi}}\left\{\frac{\partial^{2}}{\partial \tau_{l k} \partial \xi^{R}} \ln p(\boldsymbol{r} \mid \boldsymbol{\psi})\right\}=0 \\
& -E_{\boldsymbol{r} \mid \boldsymbol{\psi}}\left\{\frac{\partial^{2}}{\partial \tau_{l k} \partial \xi^{I}} \ln p(\boldsymbol{r} \mid \boldsymbol{\psi})\right\}=0 \\
& -E_{\boldsymbol{r} \mid \boldsymbol{\psi}}\left\{\frac{\partial^{2}}{\partial \Delta \phi_{k}^{t} \partial \Delta \phi_{k^{\prime}}^{t}} \ln p(\boldsymbol{r} \mid \psi)\right\}=\left\{\begin{array}{l}
0, \quad k \neq k^{\prime} \\
\frac{2 N|\xi|^{2}}{N_{0}}, \quad k=k^{\prime}
\end{array}\right. \\
& -E_{\boldsymbol{r} \mid \boldsymbol{\psi}}\left\{\frac{\partial^{2}}{\partial \Delta \phi_{k}^{t} \partial \Delta \phi_{l}^{r}} \ln p(\boldsymbol{r} \mid \boldsymbol{\psi})\right\}=\frac{2|\xi|^{2}}{N_{0}} \\
& -E_{\boldsymbol{r} \mid \boldsymbol{\psi}}\left\{\frac{\partial^{2}}{\partial \Delta \phi_{k}^{t} \partial \xi^{R}} \ln p(\boldsymbol{r} \mid \boldsymbol{\psi})\right\}=-\frac{2 N \xi^{I}}{N_{0}} \\
& -E_{\boldsymbol{r} \mid \psi}\left\{\frac{\partial^{2}}{\partial \Delta \phi_{k}^{t} \partial \xi^{I}} \ln p(\boldsymbol{r} \mid \psi)\right\}=\frac{2 N \xi^{R}}{N_{0}} \\
& -E_{\boldsymbol{r} \mid \psi}\left\{\frac{\partial^{2}}{\partial \Delta \phi_{l}^{t} \partial \Delta \phi_{l^{\prime}}^{t}} \ln p(\boldsymbol{r} \mid \boldsymbol{\psi})\right\}=\left\{\begin{array}{l}
0, \quad l \neq l^{\prime} \\
\frac{2 N|\xi|^{2}}{N_{0}}, \quad l=l^{\prime}
\end{array}\right. \\
& -E_{\boldsymbol{r} \mid \boldsymbol{\psi}}\left\{\frac{\partial^{2}}{\partial \Delta \phi_{l}^{t} \partial \xi^{R}} \ln p(\boldsymbol{r} \mid \boldsymbol{\psi})\right\}=-\frac{2 N \xi^{I}}{N_{0}} \\
& -E_{\boldsymbol{r} \mid \psi}\left\{\frac{\partial^{2}}{\partial \Delta \phi_{l}^{t} \partial \xi^{I}} \ln p(\boldsymbol{r} \mid \boldsymbol{\psi})\right\}=\frac{2 N \xi^{R}}{N_{0}} \\
& -E_{\boldsymbol{r} \mid \boldsymbol{\psi}}\left\{\frac{\partial^{2}}{\left(\partial \xi^{R}\right)^{2}} \ln p(\boldsymbol{r} \mid \boldsymbol{\psi})\right\}=\frac{2 N^{2}}{N_{0}} \\
& -E_{\boldsymbol{r} \mid \boldsymbol{\psi}}\left\{\frac{\partial^{2}}{\left(\partial \xi^{I}\right)^{2}} \ln p(\boldsymbol{r} \mid \boldsymbol{\psi})\right\}=\frac{2 N^{2}}{N_{0}} \\
& -E_{\boldsymbol{r} \mid \boldsymbol{\psi}}\left\{\frac{\partial^{2}}{\partial \xi^{R} \partial \xi^{I}} \ln p(\boldsymbol{r} \mid \psi)\right\}=0
\end{aligned}
$$

where $p(\boldsymbol{r} \mid \boldsymbol{\psi})=p(\boldsymbol{r} \mid \vartheta), l^{\prime}, k^{\prime}=1, \ldots, N$ is introduced to the different subscripts.

Meanwhile, considering that the structure of $\boldsymbol{J}(\boldsymbol{\psi})$ is relevant to the elements arrangement in $\psi$, it is convenient to divide it into the following form:

$$
\boldsymbol{J}(\boldsymbol{\psi})=\left[\begin{array}{ll}
\boldsymbol{T}_{N^{2} \times N^{2}} & \mathbf{0}_{N^{2} \times 2 N} \\
\mathbf{0}_{2 N \times N^{2}} & \boldsymbol{G}_{2 N \times 2 N}
\end{array}\right]
$$

where

$$
\begin{gathered}
\boldsymbol{T}=\frac{8 \pi^{2}|\xi|^{2}}{N_{0}} \operatorname{diag}\left(\beta_{1}^{2}, \beta_{2}^{2}, \ldots, \beta_{N}^{2}\right) \otimes \boldsymbol{I}_{N} \\
\boldsymbol{G}=\frac{2}{N_{0}}\left[\begin{array}{ccc}
\boldsymbol{\Lambda}_{(N-1) \times(N-1)} & \boldsymbol{A}^{\mathrm{T}} & \boldsymbol{F}^{\mathrm{T}} \\
\boldsymbol{A}_{(N-1) \times(N-1)} & \boldsymbol{\Lambda} & \boldsymbol{F}^{\mathrm{T}} \\
\boldsymbol{F}_{2 \times(N-1)} & \boldsymbol{F} & \boldsymbol{D}_{2 \times 2}
\end{array}\right] \\
\boldsymbol{\Lambda}=N|\xi|^{2} \boldsymbol{I}_{N-1} ; \boldsymbol{D}=N^{2} \boldsymbol{I}_{2} ; \boldsymbol{A}=|\xi|^{2} \mathbf{1}_{N-1} \\
\boldsymbol{F}=N\left[\begin{array}{c}
-\xi^{I} \\
\xi^{R}
\end{array}\right] \mathbf{1}_{1 \times N-1} .
\end{gathered}
$$$$
\beta_{k} \triangleq \sqrt{\int_{W_{k}} f^{2}\left|S_{k}(f)\right|^{2} \mathrm{~d} f / \int_{W_{k}}\left|S_{k}(f)\right|^{2} \mathrm{~d} f} \text { is defined }
$$

as the effective bandwidth of each transmitted orthogonal waveform, where the integration is over the range of frequencies with non-zero signal content $W_{k}, S_{k}(f)$ is the frequency spectrum of $s_{k}(t) ; \otimes$ represents the Kronecker product.

As the target scattering response is commonly frequency selective, orthogonal waveforms transmitted for estimating CPs are suggested to own the common frequency band to avoid introducing additional errors in CPs estimation. Therefore, we further assume all transmitted waveforms have the same band and effective bandwidth, i.e., $B=$ $B_{k}, \beta=\beta_{k}(k=1, \ldots, N)$. Then, substituting (17) $-(25)$ into (14), we have

$$
\begin{gathered}
\boldsymbol{J}^{-1}(\boldsymbol{\vartheta})=\left[\begin{array}{cc}
\boldsymbol{H} \boldsymbol{T}^{-1} \boldsymbol{H}^{\mathrm{T}} & \mathbf{0}^{\mathrm{T}} \\
\mathbf{0} & \boldsymbol{G}^{-1}
\end{array}\right]= \\
{\left[\begin{array}{cc}
\frac{N_{0}}{8 \pi^{2}|\xi|^{2} \beta^{2}} \Xi & \mathbf{0}^{\mathrm{T}} \\
\mathbf{0} & \boldsymbol{G}^{-1}
\end{array}\right]}
\end{gathered}
$$

where

$$
\Xi=\left[\begin{array}{cc}
\frac{1}{N}\left(\boldsymbol{I}_{N-1}+\mathbf{1}_{N-1}\right) & \mathbf{0}^{\mathrm{T}} \\
\mathbf{0}_{(N-1) \times(N-1)} & \frac{1}{N}\left(\boldsymbol{I}_{N-1}+\mathbf{1}_{N-1}\right)
\end{array}\right] .
$$

From (23), $\boldsymbol{G}^{-1}$ cannot be calculated directly, thus we repartition $G$ as

$$
\boldsymbol{G}=\frac{2}{N_{0}}\left[\begin{array}{cc}
\boldsymbol{\Sigma}_{2(N-1) \times 2(N-1)} & \boldsymbol{U}^{\mathrm{T}} \\
\boldsymbol{U}_{2 \times 2(N-1)} & \boldsymbol{D}_{2 \times 2}
\end{array}\right]
$$

where

$$
\boldsymbol{\Sigma}=\left[\begin{array}{cc}
\boldsymbol{\Lambda} & \boldsymbol{A}^{\mathrm{T}} \\
\boldsymbol{A} & \boldsymbol{\Lambda}
\end{array}\right], \quad \boldsymbol{U}=\left[\begin{array}{ll}
\boldsymbol{F} & \boldsymbol{F}
\end{array}\right]
$$

Usually, $\boldsymbol{\Sigma}$ and $\boldsymbol{D}$ are invertible, and one can apply the block matrix inversion lemma [26] to (28), obtaining 


$$
\boldsymbol{G}^{-1}=\frac{N_{0}}{2}\left[\begin{array}{cc}
\left(\boldsymbol{\Sigma}-\boldsymbol{U}^{\mathrm{T}} \boldsymbol{D}^{-1} \boldsymbol{U}\right)^{-1} & -\boldsymbol{\Sigma}^{-1} \boldsymbol{U}^{\mathrm{T}}\left(\boldsymbol{D}-\boldsymbol{U} \boldsymbol{\Sigma}^{-1} \boldsymbol{U}^{\mathrm{T}}\right)^{-1} \\
-\boldsymbol{D}^{-1} \boldsymbol{U}\left(\boldsymbol{\Sigma}-\boldsymbol{U}^{\mathrm{T}} \boldsymbol{D}^{-1} \boldsymbol{U}\right)^{-1} & \left(\boldsymbol{D}-\boldsymbol{U} \boldsymbol{\Sigma}^{-1} \boldsymbol{U}^{\mathrm{T}}\right)^{-1}
\end{array}\right]
$$

As $\xi^{R}$ and $\xi^{I}$ serve as nuisance parameters, it just needs to calculate the upper left submatrix $\boldsymbol{G}^{-1}$. From (24), (25) and (29), we obtain

$$
\begin{gathered}
\boldsymbol{\Sigma}-\boldsymbol{U}^{\mathrm{T}} \boldsymbol{D}^{-1} \boldsymbol{U}=\left[\begin{array}{cc}
\boldsymbol{\Lambda} & \boldsymbol{A}^{\mathrm{T}} \\
\boldsymbol{A} & \boldsymbol{\Lambda}
\end{array}\right]-\frac{1}{N^{2}}\left[\begin{array}{c}
\boldsymbol{F}^{\mathrm{T}} \\
\boldsymbol{F}^{\mathrm{T}}
\end{array}\right]\left[\begin{array}{ll}
\boldsymbol{F} & \boldsymbol{F}
\end{array}\right]= \\
{\left[\begin{array}{cc}
\boldsymbol{\Lambda}-|\xi|^{2} \mathbf{1}_{N-1} & \boldsymbol{O}^{\mathrm{T}} \\
\boldsymbol{O}_{(N-1) \times(N-1)} & \boldsymbol{\Lambda}-|\xi|^{2} \boldsymbol{1}_{N-1}
\end{array}\right] .}
\end{gathered}
$$

Applying the Woodbury matrix inverse lemma [26] to (31), its inverse can be derived as

$$
\begin{gathered}
\left(\boldsymbol{\Sigma}-\boldsymbol{U}^{\mathrm{T}} \boldsymbol{D}^{-1} \boldsymbol{U}\right)^{-1}= \\
\frac{1}{|\xi|^{2}}\left[\begin{array}{cc}
\frac{1}{N}\left(\boldsymbol{I}_{N-1}+\mathbf{1}_{N-1}\right) & \boldsymbol{O} \\
\boldsymbol{O} & \frac{1}{N}\left(\boldsymbol{I}_{N-1}+\mathbf{1}_{N-1}\right)
\end{array}\right] .
\end{gathered}
$$

Now, all necessary elements in $\boldsymbol{J}^{-1}(\boldsymbol{\vartheta})$ related to CPs $\mathrm{CRB}$ are presented in (26), (27) and (32), and therefore the variances of CPs estimate are bounded by

$$
\begin{gathered}
\operatorname{var}\left(\Delta \widehat{\tau}_{k}^{t}\right) \geqslant\left[\boldsymbol{J}^{-1}(\boldsymbol{\vartheta})\right]_{k-1, k-1}=\frac{1}{4 \pi^{2} \beta^{2} \mathrm{SNR}_{\mathrm{CRB}} N} \\
\operatorname{var}\left(\Delta \widehat{\tau}_{l}^{r}\right) \geqslant\left[\boldsymbol{J}^{-1}(\boldsymbol{\vartheta})\right]_{N+l-2, N+l-2}=\frac{1}{4 \pi^{2} \beta^{2} \mathrm{SNR}_{\mathrm{CRB}} N} \\
\operatorname{var}\left(\Delta \widehat{\phi}_{k}^{t}\right) \geqslant\left[\boldsymbol{J}^{-1}(\boldsymbol{\vartheta})\right]_{2 N+k-3,2 N+k-3}=\frac{1}{\operatorname{SNR}_{\mathrm{CRB}} N} \\
\operatorname{var}\left(\Delta \widehat{\phi}_{l}^{r}\right) \geqslant\left[\boldsymbol{J}^{-1}(\boldsymbol{\vartheta})\right]_{3 N+l-4,3 N+l-4}=\frac{1}{\mathrm{SNR}_{\mathrm{CRB}} N}
\end{gathered}
$$

where $\left\{\Delta \widehat{\tau}_{k}^{t}, \Delta \widehat{\tau}_{l}^{r}, \Delta \widehat{\phi}_{k}^{t}, \Delta \widehat{\phi}_{l}^{r}\right\}(l, k=2, \ldots, N)$ are the CPs estimates and $\mathrm{SNR}_{\mathrm{CRB}}=|\xi|^{2} / N_{0}$.

Here, we explain the definition of $\mathrm{SNR}_{\mathrm{CRB}}$. In practice, the PSD of the white noise is band-limited. It exists only in the frequency band with the same range as the sampling bandwidth, i.e., $N_{0}=\sigma_{w}^{2} / f_{s}$, where $\sigma_{w}^{2}$ is the power of the white noise, and $f_{s}$ is the sampling frequency. Since the energy of the baseband signal is assumed to be normalized, the SNR of the single radar before matched filtering is $\mathrm{SNR}_{\text {in }}=|\xi|^{2} / T \sigma_{w}^{2}$. And the SNR after matched filtering is $\mathrm{SNR}_{\mathrm{MF}}^{\mathrm{in}}=B T \cdot \mathrm{SNR}_{\mathrm{in}}=|\xi|^{2} B / \sigma_{w}^{2}, B$ is the bandwidth of the baseband signal. Obviously, only $f_{s}=B, \mathrm{SNR}_{\mathrm{CRB}}=\mathrm{SNR}_{\mathrm{MF}}^{\mathrm{in}}$. Otherwise, $\mathrm{SNR}_{\mathrm{MF}}^{\mathrm{in}}=$ $\mathrm{SNR}_{\mathrm{CRB}}+10 \ln \left(B / f_{s}\right)$.

As the maximum likelihood estimates (MLE) can be closely approached to the CRB under asymptotic conditions, i.e., sufficiently large SNR or observation time [27]. Thus we use it to verify the correctness of the CRB we have derived. Focus on a dual-radar coherently combining scenario in the white Gaussian noise. Radar 1 and radar 2 are located at $(-d / 2,0)$ and $(d / 2,0)$ respectively, where $d=12 \mathrm{~m}$. To obtain ideally orthogonal signals, the TDM technique is used at the radar transmitter. Both radars transmit the chirp signal, chirp duration $T_{p}=1 \mu \mathrm{s}$, bandwidth $B=300 \mathrm{MHz}, \beta^{2}=B^{2} / 12$ [28]. The target is located at $\left(R_{0} \cos \theta, R_{0} \sin \theta\right), R_{0}=200 \mathrm{~km}, R_{0} \gg d, \theta=60^{\circ}$. Other used parameters are listed in the following: carrier frequency $f_{c}=10 \mathrm{GHz}$, sampling frequency $f_{s}=1 \mathrm{GHz}$ and the synchronization error $\kappa_{k}=\phi_{k}=0(k=1,2)$. The root mean square error (RMSE) of the MLE are evaluated by using MC simulations with 200 iterations per $\mathrm{SNR}_{\mathrm{MF}}^{\mathrm{in}}$ value. Simulation results are shown in Fig. 4.
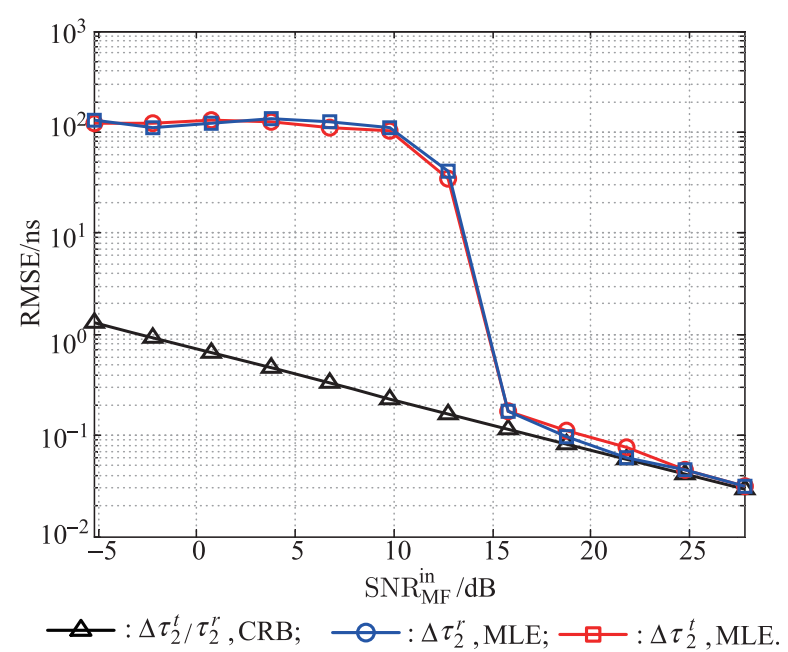

(a) Time CPs

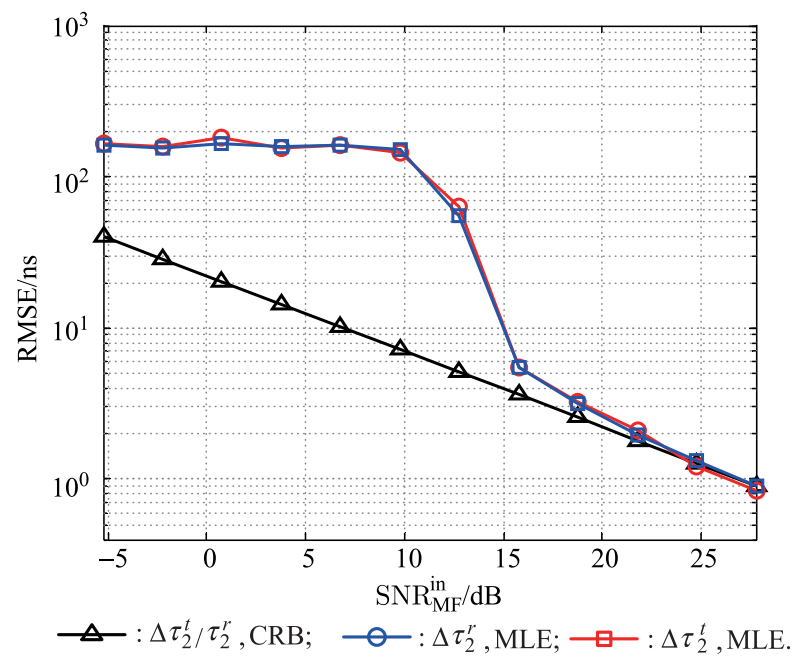

(b) Phase CPs

Fig. 4 RMSE of the CRB versus the computed MLE for a dual radar coherently combining case 
From (33) and Fig. 4, we make the following observations:

(i) The RMSE of CPs MLE approaches the CRB arbitrarily close in high $\mathrm{SNR}_{\mathrm{MF}}^{\mathrm{in}}$, which verifies the correctness of the CRB we have derived.

(ii) The CRB of time CPs is inversely proportional to the square of the effective bandwidth, the number of radars and $\mathrm{SNR}_{\mathrm{MF}}^{\mathrm{in}}$. While for the phase CPs CRB, they are only inversely proportional to the number of radars and $\mathrm{SNR}_{\mathrm{MF}}^{\mathrm{in}}$. Moreover, both of them are independent of the carrier frequency.

\section{Performance gain bounds}

As aforementioned in Section 2, once CPs estimates are obtained, all radars will transmit the identical waveform $s(t)$. Signal decoherence among radars is calibrated by carrying out transmitting/receiving time and phase adjustments via CPs estimates. Next, we will discuss the impact of the uncertainty of CPs estimates on coherently combing performance.

\subsection{Evaluation metric of performance gain}

After carrying out the transmitting time and phase adjustments, all radars' transmitted signals will coherently superimpose at the target to achieve the coherent-on-transmit. The superimposed signal can be expressed as follows:

$$
\sum_{k=1}^{N} s\left(t-\tau_{1}+\delta \Delta \tau_{k}^{t}-\kappa_{1}\right) \mathrm{e}^{-j \delta \Delta \phi_{k}^{t}} \mathrm{e}^{j 2 \pi f_{c}\left(t-\tau_{1}-\kappa_{1}\right)} \mathrm{e}^{j \phi_{1}}
$$

where $\delta \Delta \tau_{k}^{t}=\Delta \widehat{\tau}_{k}^{t}-\Delta \tau_{k}^{t}$ and $\delta \Delta \phi_{k}^{t}=\Delta \widehat{\phi}_{k}^{t}-\Delta \phi_{k}^{t}$ represent the transmit CPs estimation residuals. Especially, $\delta \Delta \tau_{1}^{t}=\delta \Delta \phi_{1}^{t}=0$, as reference radar (radar 1) does not require time and phase adjustment.

Then, all radars can receive the echo of this superimposed signal impinging on the target. Similarly, after adjusting the time and phase of received echoes, the coherenton-transmit can be achieved. The ultimate coherently combining signal is (coherent-on-transmit and -receive)

$$
\begin{gathered}
r(t)=\xi \sum_{l=1}^{N} \sum_{k=1}^{N} s\left(t-\tau_{11}+\delta \Delta \tau_{k}^{t}+\right. \\
\left.\delta \Delta \tau_{l}^{r}\right) \mathrm{e}^{-j \delta \Delta \phi_{k}^{t}} \mathrm{e}^{-j \delta \Delta \phi_{l}^{r}}+\sum_{l=1}^{N} w_{l}(t)
\end{gathered}
$$

where $\delta \Delta \tau_{l}^{r}=\Delta \widehat{\tau}_{l}^{r}-\Delta \tau_{l}^{r}, \delta \Delta \phi_{l}^{r}=\Delta \widehat{\phi}_{l}^{r}-\Delta \phi_{l}^{r}$ represent the receive CPs estimation residuals. Similarly, $\delta \Delta \tau_{1}^{r}=\delta \Delta \phi_{1}^{r}=0$.
Analyzing from (35) is not a convenient approach to evaluate the performance gain. In practice, before further processing, echoes are often processed by the matched filtering firstly. Then the energy of the echo is concentrated at the peak of the pulse compression output, and such processing help us to analyze the performance gain.

From (35), the peak of the pulse compression output related to $r(t)$ is

$$
\begin{gathered}
S_{\mathrm{MF}}\left(\tau_{11}\right)=\xi \sum_{l=1}^{N} \sum_{k=1}^{N} p\left(\delta \Delta \tau_{k}^{t}+\right. \\
\left.\delta \Delta \tau_{l}^{r}\right) \mathrm{e}^{-j \delta \Delta \phi_{k}^{t}} \mathrm{e}^{-j \delta \Delta \phi_{l}^{r}}+\sum_{l=1}^{N} w_{l \mathrm{MF}}\left(\tau_{11}\right)
\end{gathered}
$$

where $p(t)=s(t) * s^{*}(-t), w_{l \mathrm{MF}}(t)=w_{l}(t) * s^{*}(-t)$, $*$ is the convolution operation. Note that, $w_{l \mathrm{MF}}(t)$ is a zero mean, Gaussian random process, whose variance is $\delta_{w}^{2}=\int_{-\infty}^{+\infty} N_{0}\left|S^{*}(f)\right|^{2} \mathrm{~d} f=N_{0}$.

In (36), it can be found that the uncertainty of CPs estimates directly affects the intensity of the pulse compression output peak. In Section 4, we derive the theoretical bound to characterize the estimation uncertainty, i.e., the CRB. Based on this bound and (36), it is available to analyze the performance gain under the given CPs estimation bound.

As we know, the CRB is the bound in the statistical sense. The estimation uncertainty it represents should be presented in the form of statistics. For simplicity, CPs estimation residuals are assumed to be independent with the Gaussian distribution. Take $\delta \Delta \tau_{k}^{t}$ as an example, it obeys $N\left(0, \sigma_{\Delta \widehat{\tau}_{k}^{t}, \mathrm{CRB}}^{2}\right), \sigma_{\Delta \widehat{\tau}_{k}^{t}, \mathrm{CRB}}^{2}$ is the CRB derived in (33). Under this assumption, we can calculate the average power of the signal part at the peak output $S_{\mathrm{MF}}\left(\tau_{11}\right)$ under the given CPs estimation bound, which is

$$
\begin{gathered}
P_{\text {co }}=|\xi|^{2} E_{\delta \Delta \phi_{k}^{t}, \delta \Delta \phi_{k^{\prime}}^{t}, \delta \Delta \phi_{l}^{r}, \delta \Delta \phi_{l^{\prime}}^{r}}\left[\sum_{l=1}^{N} \sum_{k=1}^{N} \sum_{l^{\prime}=1}^{N} \sum_{k^{\prime}=1}^{N} .\right. \\
\mathrm{e}^{-j\left(\delta \Delta \phi_{k}^{t}-\delta \Delta \phi_{k^{\prime}}^{t}\right)} \mathrm{e}^{-j\left(\delta \Delta \phi_{l}^{r}-\delta \Delta \phi_{l^{\prime}}^{r}\right)} \cdot p\left(\delta \Delta \tau_{k}^{t}+\right. \\
\left.\left.\delta \Delta \tau_{l}^{r}\right) p\left(\delta \Delta \tau_{k^{\prime}}^{t}+\delta \Delta \tau_{l^{\prime}}^{r}\right)\right] .
\end{gathered}
$$

For conciseness, in the following, $E[\cdot]$ refers to taking the expectation for $\delta \Delta \phi_{k}^{t}, \delta \Delta \phi_{k^{\prime}}^{t}, \delta \Delta \phi_{l}^{r}, \delta \Delta \phi_{l^{\prime}}^{r}$.

To quantitatively evaluate the performance gain brought by the multi-radar coherently combination, we introduce the average SNR gain (SNRg), which is defined as follows:

$$
\mathrm{SNRg}=\frac{\mathrm{SNR}_{\mathrm{MF}}^{\mathrm{co}}}{\mathrm{SNR}_{\mathrm{MF}}^{\text {in }}}=\frac{P_{\mathrm{co}} / N N_{0}}{|\xi|^{2} / N_{0}}=
$$




$$
\begin{gathered}
\frac{1}{N} E\left[\sum_{l=1}^{N} \sum_{k=1}^{N} \sum_{l^{\prime}=1}^{N} \sum_{k^{\prime}=1}^{N} \mathrm{e}^{-j\left(\delta \Delta \phi_{k}^{t}-\delta \Delta \phi_{k^{\prime}}^{t}\right)} \mathrm{e}^{-j\left(\delta \Delta \phi_{l}^{r}-\delta \Delta \phi_{l^{\prime}}^{r}\right) .}\right. \\
\left.p\left(\delta \Delta \tau_{k}^{t}+\delta \Delta \tau_{l}^{r}\right) p\left(\delta \Delta \tau_{k^{\prime}}^{t}+\delta \Delta \tau_{l^{\prime}}^{r}\right)\right]
\end{gathered}
$$

where $\mathrm{SNR}_{\mathrm{MF}}^{\mathrm{in}}$ is the $\mathrm{SNR}$ for the single radar.

The CRB provides the lower bound for the estimation. It characterizes the optimal estimation performance under the given measurement noise. Therefore, the calculated SNRg in (38) is the theoretically achievable optimal gain, i.e., the upper performance gain bound.

Considering that $p(t)$ in (37) is usually a nonlinear function, it is difficult to derive the closed form of the SNRg. Therefore, two cases are analyzed in the following.

\subsection{Non-tight closed upper bound}

If time CPs are ideally compensated, the SNRg is independent of $p(t),(38)$ becomes

$$
\begin{gathered}
\mathrm{SNRg}^{\text {phase }}=\frac{1}{N} E\left[\sum_{l=1}^{N} \sum_{k=1}^{N} \sum_{l^{\prime}=1}^{N} \sum_{k^{\prime}=1}^{N} .\right. \\
\left.\mathrm{e}^{-j\left(\delta \Delta \phi_{k}^{t}-\delta \Delta \phi_{k^{\prime}}^{t}\right)} \mathrm{e}^{-j\left(\delta \Delta \phi_{l}^{r}-\delta \Delta \phi_{l^{\prime}}^{r}\right)}\right]= \\
\frac{1}{N}\left[2(N-1) \mathrm{e}^{-\frac{1}{2 N \mathrm{SNR} C R B}}+N+\right. \\
(N-1)(N-2) \mathrm{e}^{\left.-\frac{1}{N \mathrm{SNR} C \mathrm{CRB}}\right]^{2} .}
\end{gathered}
$$

Here, SNRg is denoted as SNRg ${ }^{\text {phase }}$ for the distinction.

In practice, time CPs cannot be compensated ideally, thus the following relationship holds

$$
\mathrm{SNRg}<\mathrm{SNRg}{ }^{\text {phase }} .
$$

That is, (39) presents a non-tight upper bound of the SNRg.

\subsection{Tight upper bound based on Monte Carlo simulation}

Particularly, we assume $s(t)$ is a linear frequency modulation (LFM) signal with large time-bandwidth product, $p(t) \approx \sin c\left(\pi B_{\mathrm{FC}} t\right)$ (energy of the LFM is normalized), $B_{\mathrm{FC}}$ is the bandwidth of the LFM, then (38) becomes

$$
\begin{gathered}
\operatorname{SNRg}=\frac{1}{N} \sum_{l=1}^{N} \sum_{k=1}^{N} \sum_{l^{\prime}=1}^{N} \sum_{k^{\prime}=1}^{N} . \\
E\left[\mathrm{e}^{-j\left(\delta \Delta \phi_{k}^{t}-\delta \Delta \phi_{k^{\prime}}^{t}\right)} \mathrm{e}^{-j\left(\delta \Delta \phi_{l}^{r}-\delta \Delta \phi_{l^{\prime}}^{r}\right)}\right] \cdot \Omega
\end{gathered}
$$

where

$$
\Omega=E\left[\sin c\left(\pi B_{\mathrm{FC}}\left(\delta \Delta \tau_{k}^{t}+\delta \Delta \tau_{l}^{r}\right)\right) .\right.
$$

$$
\left.\sin c\left(\pi B_{\mathrm{FC}}\left(\delta \Delta \tau_{k^{\prime}}^{t}+\delta \Delta \tau_{l^{\prime}}^{r}\right)\right)\right] .
$$

$\forall l, k, l, k \in\{1,2, \ldots, N\}$, the random variable $\Theta=$ $\delta \Delta \tau_{k}^{t}+\delta \Delta \tau_{l}^{r}$ subjects to the following distribution:

$$
\Theta \sim\left\{\begin{array}{l}
0, \quad k=l=0 \\
\boldsymbol{N}\left(0, \sigma_{\Delta \widehat{\tau}}^{2}\right), \quad k=0 \vee l=0 \\
\boldsymbol{N}\left(0,2 \sigma_{\Delta \widehat{\tau}}^{2}\right), \quad \text { others }
\end{array}\right.
$$

where $\sigma_{\Delta \widehat{\tau}}^{2}=1 / 4 \pi^{2} \beta^{2} \operatorname{SNR}_{\mathrm{CRB}} N$. For conciseness, (42) can be further expressed as $\Theta \sim \boldsymbol{N}\left(0, \pi^{2} B_{\mathrm{FC}}^{2} a \sigma_{\Delta \widehat{\tau}}^{2}\right)$, $a \in\{0,1,2\}$.

Generally, for orthogonal waveforms, their effective bandwidth can be approximated to $\beta=2.72 B / 2 \pi$ [28], $B$ is the bandwidth of orthogonal signals. Thus

$$
\Theta \sim N\left(0, \frac{1}{14.7968} \frac{B_{\mathrm{FC}}^{2}}{B^{2}} \frac{a \pi^{2}}{\operatorname{SNR}_{\mathrm{CRB}} N}\right) .
$$

Specifically, from (43), if $B=B_{\mathrm{FC}}, \Theta$ is independent of $B$. That is, if the orthogonal waveforms used for CPs estimation and the identical waveform used for coherently combining have the same bandwidth, the SNRg bound is independent of the bandwidth.

From (41) and (43), it is hard to give the bound in the closed-form; hence we use MC simulations to approximate it. Meanwhile, we also implement the same simulations to approximate $\mathrm{SNRg}^{\text {phase }}$ as the comparison for theoretical derivation results in (39) to verify the validity of the MC simulations.

Consider a dual-radar coherently combining scenario, $B=300 \mathrm{MHz}, B_{\mathrm{FC}}=100 \mathrm{MHz}$ and $300 \mathrm{MHz}$ respectively. In each $\mathrm{SNR}_{\mathrm{CRB}}$ step, we run 200 iterations. Then change the number of radars to 6 , and repeat the simulation. Results are shown in Fig. 5.

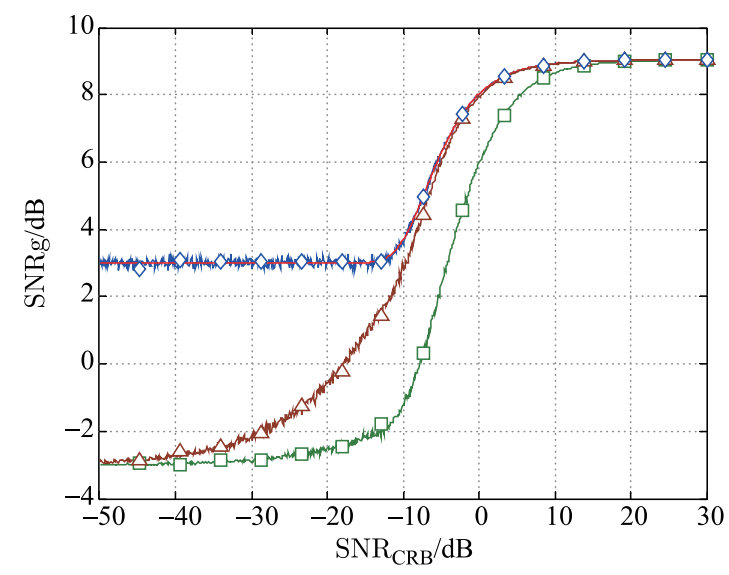

(a) $\mathrm{N}=2$ 


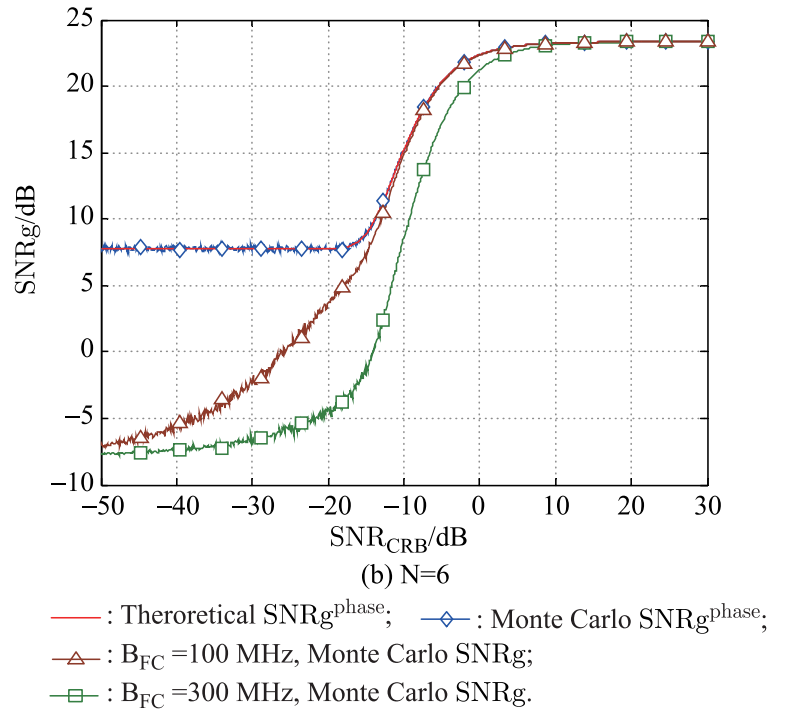

Fig. 5 Bound of SNRg calculated via Monte Carlo simulation and theoretical derivation under different $\mathrm{SNR}_{\mathrm{CRB}}$ and $B_{\mathrm{FC}}$

From Fig. 5, we can make the following observations:

(i) The non-tight upper bound derived in (39), marked as Theoretical SNRg phase in Fig. 5, coincides with the result of the MC simulation, which verifies the validity of the $\mathrm{MC}$ simulation.

(ii) As $\mathrm{SNR}_{\mathrm{CRB}}$ increases, $\mathrm{SNRg}$ increases, and vice versa. When $\mathrm{SNR}_{\mathrm{CRB}} \rightarrow+\infty, \mathrm{SNRg} \rightarrow N^{3}$, the optimal SNRg is obtained. When $\mathrm{SNR}_{\mathrm{CRB}} \rightarrow-\infty, \mathrm{CPs}$ cannot be accurately estimated, SNRg $\rightarrow 1 / N$, the minimum SNRg is obtained. Particularly, if $B_{\mathrm{FC}}=B, \mathrm{SNRg}^{\text {phase }} \approx$ SNRg conditioned on SNRg $>$ N.

(iii) For a certain $\mathrm{SNR}_{\mathrm{CRB}}$ and $B$, the narrower $B_{\mathrm{FC}}$ is, the higher SNRg can be obtained. Furthermore, comparing Fig. 5(a) and Fig. 5(b), it can be found that increasing the number of radars will be benefitial to obtaining the coherent gain under the same noise conditions.

\subsection{Discussion}

According to the performance bound obtained above, the following comments are intended to provide some useful conclusions:

(i) The SNR benchmark. The upper bounds of SNRg depend on the SNR of the single radar, only it above a certain benchmark, considerable SNRg can be obtained. Otherwise, the anticipant performance gain cannot be obtained. Particularly, considering a large array radar with the fixed number of array elements, if we uniformly divide it into several parts, and let each part act as an independent radar. Interestingly, the more parts we divide, the more unfavorable to obtain the considerable SNR gain. The reason lies that such division leads to a sharp SNR dropping for each small separated radar. The SNRg loss caused by the SNR dropping is far more than the SNRg benefit obtained by the increased number of radars. This implies that the size of the radar to be combined should not be too small.

(ii) Insightful suggestions. Two feasible approaches can increase the upper bound of the SNRg under the given noise conditions, i.e. adding the number of the radar, and using wideband orthogonal waveforms to estimate CPs for coherently combining radars transmitting narrower band waveform.

\section{Conclusions}

Coherently combining multiple radars in a target-based calibration manner is an appealing technology that achieves the considerable coherent gain while providing transportability and economic advantage. The targetbased manner is essentially a cognitive process [29,30]. By transmitting orthogonal waveforms, multiple radars perceive the decoherence among radars dynamically to obtain the necessary information, i.e., CPs, for the decoherence calibration. However, such perception exists uncertainty, which will affect the performance gain after multi-radar coherent combination. In this paper, we propose the theoretical bound characterizing the uncertainty in perception on the basis of the given signal probability model. Based on the CPs CRB, we further propose the theoretically achievable upper performance gain bound, including the nontight bound and the tight upper bound. From these upper bounds, it can be found that in order to obtain the considerable performance gain from multi-radar coherent combination via the target-based calibration manner, the SNR of the single radar should not be too low. Therefore, these bounds can be used to guide multiple radars coherent combination in practice, especially for designing the unit radars to be combined.

\section{References}

[1] THOME G D, ROBERT P E, STEUDEL F. System and method for coherently combining a plurality of radars. U.S. Patent, 2008.

[2] FLETCHER A S, ROBEY F C. Performance bounds for adaptive coherence of sparse array radar. Proc. of the 11th Conference Adaptive Sensors Array Processing, 2003: 1-6.

[3] CUOMO K M, COUTTS S D, MCHARG J C, et al. Wideband aperture coherence processing for next generation radar (NexGen). Lexington: MIT Lincoln Laboratory, 2004: 11-16.

[4] BROOKNER E, MANOOGIAN D V, STEUDEL F. Multiple radar combining for increased range, radar sensitivity and angle accuracy. EP, US6977610.

[5] COUTTS S, CUOMO K, MCHARG J, et al. Distributed coherent aperture measurements for next generation BMD radar. Proc. of the 4th IEEE Workshop on Sensor Array and Multichannel Processing, 2006: 390-393.

[6] GRIFFITHS H D, BAKER C, ADAMY D. Stimson's introduction to airborne radar. Hertfordshire: Scitech Pub Incorporated, 2014. 
[7] LIU X, XU Z, LIU X, et al. A clean signal reconstruction approach for coherently combining multiple radars. EURASIP Journal on Advances in Signal Processing, 2018, 2018(1): 47 57.

[8] LIU X, XU Z, WANG L, et al. Dual-radar coherently combining: generalized paradigm and verification example. IET Radar, Sonar \& Navigation, 2019, 13, (5): 689-699.

[9] ZENG T, YIN P, LIU Q. Wideband distributed coherent aperture radar based on stepped frequency signal: theory and experimental results. IET Radar, Sonar \& Navigation, 2016, 10(4): $672-688$.

[10] YIN P, YANG X, LIU Q, et al. Wideband distributed coherent aperture radar. Proc. of the IEEE Radar Conference, 2014: $1114-1117$.

[11] YIN P, YANG X, ZENG T, et al. Robust time synchronization method based on step frequency signal for wideband distributed coherent aperture radar. Proc. of the IEEE International Symposium on Phased Array Systems and Technology, 2013: $383-388$.

[12] GAO H, CAO Z, WEN S, et al. Study on distributed aperture coherence-synthesizing radar with several experiment results. Proc. of the IEEE CIE International Conference on Radar, 2011: $84-86$

[13] GAO H, CAO Z, LU Y. Development of distributed aperture coherence-synthetic radar technology. Proc. of the IET International Radar Conference, 2013: 1-5.

[14] GAO H, ZHOU B, ZHOU D, et al. Performance analysis and experimental study on distributed aperture coherencesynthetic radar. Proc. of the CIE International Conference on Radar, 2016: 1-5.

[15] SUN P, TANG J, HE Q, et al. Cramer-Rao bound of parameters estimation and coherence performance for next generation radar. IET Radar, Sonar \& Navigation, 2013, 7(5): 553-567.

[16] SUN P, TANG J, TANG X. Cramer-Rao bound and signal-tonoise ratio gain in distributed coherent aperture radar. Journal of Systems Engineering and Electronics, 2014, 25(2): $217-$ 225.

[17] LEHMANN N H, FISHLER E, HAIMOVICH A M, et al. Evaluation of transmit diversity in MIMO-radar direction finding. IEEE Trans. on Signal Processing, 2007, 55(5): $2215-$ 2225.

[18] LIU W, WANG Y, LIU J, et al. Adaptive detection without training data in colocated MIMO radar. IEEE Trans. on Aerospace and Electronic Systems, 2015, 51(3): 2469-2479.

[19] VAN T H, BELL H. Bayesian Bounds for Parameter Estimation and Nonlinear Filtering/Tracking. New York: Wiley-IEEE Press, 2007.

[20] HE Q, BLUM R S, GODRICH H, et al. Target velocity estimation and antenna placement for MIMO radar with widely separated antennas. IEEE Journal of Selected Topics in Signal Processing, 2010, 4(1): 79-100.

[21] LIU W, WANG Y, XIE W. Fisher information matrix, Rao test, and Wald test for complex-valued signals and their applications. Signal Processing, 2014, 94: 1-5.

[22] GODRICH H, HAIMOVICH A M, BLUM R S. Target lo- calization accuracy gain in MIMO radar-based systems. IEEE Trans. on Information Theory, 2010, 56(6): 2783-2803.

[23] LEHMANN N H, HAIMOVICH A M, BLUM R S, et al. High resolution capabilities of mimo radar. Proc. of the 40th Asilomar Conference on Signals, Systems and Computers, 2006: $25-30$.

[24] POOR H V. An introduction to signal detection and estimation. New York: Dowden \& Culver, 1994.

[25] KAY S M. Fundamentals of statistical signal processing Volume I: estimation theory. London: Pearson Education, 1993.

[26] ZHANG X D. Matrix analysis and application. Beijing: Tsinghua University Press, 2004.

[27] NOAM Y, MESSER H. Notes on the tightness of the hybrid Cramér-Rao lower bound. IEEE Trans. on Signal Processing, 2009, 57(6): 2074-2084.

[28] SKOLNIK M I. Introduction to radar system. New York: McGraw-Hill, 2002.

[29] GRECO M S, GINI F, STINCO P, et al. Cognitive radars: on the road to reality: progress thus far and possibilities for the future. IEEE Signal Processing Magazine, 2018, 35(4): $112-$ 125.

[30] HAYKIN S. Cognitive radar: a way of the future. IEEE signal processing magazine, 2006, 23(1): 30-40.

\section{Biographies}

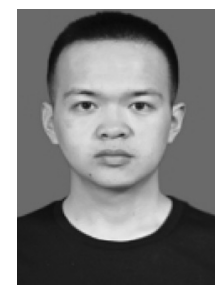

LIU Xinghua was born in 1993. He received his B.E. degree from Xidian University in 2012. In 2015, he received his bachelor's degree from Electronic Engineering Institute. Now he is a Ph.D. candidate in National University of Defense Technology (NUDT). His research interests are array signal processing and radar cooperative detection technology.

E-mail: xinghua217@163.com

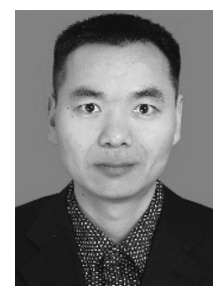

XU Zhenhai was born in 1977. He received his B.E. and Ph.D. degrees from the National University of Defense Technology (NUDT) in 1998 and 2004, respectively. He is currently a professor at NUDT. His research interests are array signal processing and radar polarimetry.

E-mail: drxzh930@sina.com

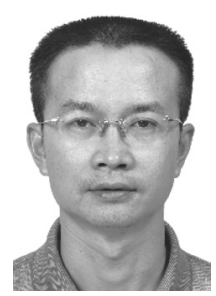

XIAO Shunping was born in 1964. He received his B.E. and Ph.D. degrees in electronic engineering from the National University of Defense Technology (NUDT) in 1986 and 1995, respectively. He is currently a professor in NUDT. His research interests are radar polarimetry, radar array signal processing, SAR and target recognition.

E-mail: xiaoshunping_nudt@163.com 